\section{A CASE OF INGUINAL ANEURISM,}

FOR THE CURE OF WHICH THE EXTERNAL ILIAC ARTERY WAS TIED.

\section{BY THE LATE SIR ASTLEY COOPER.}

THE ligature of the external iliac artery in cases of inguinal aneurism has been now so often repeated, and with so much success, that the ordinary reports of such cases present but seldom any unusual interest. The following, however, is a detailed report, by Sir Astley Cooper, of one of his earliest cases, having occurred in his practice nearly forty years ago, (a comparatively short period after the introduction of the Hunterian method of operating for aneurism;) and is also interesting, as it appears to have led that distinguished surgeon to the practice which he afterwards adopted, in opposition to that recommended by Abernethy and others ; namely, of using only one ligature, and consequently not dividing the artery. The less amount of disturbance and irritation caused by this latter method, the late improvements in surgical science, and our more accurate ideas regarding the anatomy and diseases of arteries, and, above all, an ample amount of experience, have thoroughly proved the safety and sufficiency of the single ligature, and it is now therefore adopted by all the principal operators of the present day.

William Cowles, æt. 39, a gardener by trade, about six months ago walked five miles with a heavy burden, which strained and fatigued him very much. 'Two weeks afterwards, feeling a slight pain in his groin, he examined and found a hard lump the size of a hazel-nut, which throbbed under the finger. As the pain was then inconsiderable, he disregarded it for six weeks, when the tumor had grown to the size of a large marble. He observed now, upon drinking more than usual, that the throbbing became much stronger, and the pain from tension much greater. He continued to work at his business, suffering, however, great inconvenience and pain from the tumor, more especially when he stooped. At the end of another six weeks, the swelling was as large as a walnut, beating with increased force, and in six weeks more had attained the size of a pullet's egg. He now left off work, on account of the violence of the pain, and in the following six weeks the tumor grew with increased rapidity, the pulsation became very strong, and the patient's sufferings extreme. Three weeks ago he came up to town, a distance of 120 miles, on the outside of a coach, and falling asleep on his belly, the tumor was exposed to considerable injury, and three days after his arrival, for the first time assumed on the surface a different colour from the surrounding parts. At this time he applied to me, (having come to town for that purpose). The tumor appeared then with livid spots, the patient still continuing to walk about, going twice from the Borough to Brompton, before the operation.

In this case the tumor was situated so high in the thigh as to reach and raise Poupart's ligament - the artery had dilated to nearly the size of a small pint bowl-the skin covering it had become extremely thin, tense, and irregular on its surface, and the more prominent parts were of a mixed colour, purple and red of various shades. From the unfortunately far advanced state of the disease, I considered it my duty to lose no time in performing the only operation which promised relief to the patient, which I accordingly did at once.

June 22.-The tumor was now hard, and its pulsation distinct and forcible-the thigh and leg were of a natural temperature, and exhibited no symptoms of deviation from the healthy state. Before beginning the operation, in order to provide for any untoward event that might occur, I had a large key procured, and the ring of it wrapt round with tow. With this I was able readily to compress the artery as it passes over the pubis.

I began my incision about an inch and a half from the spine of the ilium, extending it obliquely to the femoral ligament. I first cut through the integuments covering the external oblique muscle, and displayed its shining tendinous fibres. I then carefully divided these, and exposed the lower portion of the internal oblique, which, together with the inferior edge of the transversalis, I turned up with my fingers, insinuating them at the same time under the peritoneum. I was now able to feel distinctly the pulsation of the external iliac artery, and proceeded to separate it from its accompanying vein, which I did with the handle of my scalpel. From the depth of the artery, and the impossibility of distinguishing it with the eye at the bottom of the wound, I considered this to be the most difficult part of the operation. This, however, being accomplished, with a little dissection, (still with the handle of my knife,) and by dividing with a bistoury a few of the lower fibres of the internal iliac and transversalis muscles, I completely exposed the artery detached from the surrounding parts, passed a double ligature under it by means of an aneurismal needle, and after separating the two ligatures, I tied the lower one as far down the vessel as I could draw it. The pulsation in the tumor at once ceased. I could now deliberately insulate a sufficient portion of the artery to admit of the upper ligature being tied, leaving a space of about three-quarters of an inch between the two. The end of the upper ligature, to which a needle was attached, I passed through the artery, securing it by tying it with its fellow. The concluding step in the operation was the division of the artery between the ligaturesand this portion of the vessel, it may be well to say, appeared to be perfectly healthy. The external wound was now closed by two stitches, supported by intervening slips of adhesive plaster, and these were lightly covered with a fold of lint, while over all were brought the ends of a $T$ bandage, and loosely connected so as to retain it.

Towards the conclusion of the operation, the temperature of the limb became sensibly diminished, but very soon regained a degree of warmth little below the natural.

The patient was put to bed at about half-past one P.M. ; he was laid on his left side, with a pillow between his knees supporting the aneurismal thigh, (the right thigh,) and a cotton stocking was put on the right leg, and over this a doubling of thick flannel 
which extended over the knee. These measures were adopted in compliance with my orders. The warmth of the thigh I could not perceive at this time to differ from that of the other. The pulse was a little hurried from the agitation necessarily dependent on the operation, and the patient, on being questioned, said he was not sensible of greater torpor or coldness in one limb than in the other. him.

3 P.M. The patient nearly as when I last saw

4 P.м. Pulse moderate. Thigh, leg, and foot increased in warmth.

8 P.M. I called to see my patient; pulse about 85 ; foot and leg perspiring; patient free from pain. I think the tumor is not so dark coloured.

Half-past 10 P.M. I have just left my patient, who says his limb is quite comfortable; the warmth of it is about the natural standard.

June 23rd, 6 A.м. Patient has slept but little, complains of soreness of the abdomen above the wound ; pulse more full than last evening; heat of extremity natural.

10 A.M. Patient as at six.

I P.M. I saw the patient, who still complained of soreness above the wound. Sulphas sodæ ordered.

10 P.M. Salts not producing the desired effect, the senna electuary was given, but has not yet operated.

Half-past 10 P.м. Called and advised, if evacuations are not produced by the means already used before 12 P.M. that a clyster shall be administered. Pulse 96. I think the tumor is diminished.

June 24th, 6 A.м. The clyster was given as directed, and the patient had two copious evacuations during the night, and afterwards slept comfortably. Pain above the wound diminished, heat of the limb natural, pulse less full than last night.

2 P.M. Pulse fuller and stronger.

8 and 10 P.M. No alteration.

June 25th, A.M. Patient complaining much of want of rest; says that he did not sleep a quarter of an hour all night; has had no evacuation from the bowels since yesterday; pain above the wound much abated; pulse full.

3 P.M. I called and ordered the senna electuary; patient has had no evacuation; pulse full and strong.

10 P.M. Pulse 108; first perceived a discharge of pus from the wound; great constitutional irritation ; no motion yet.

26. -7 A.м. Patient delirious during the night. He required watching to prevent his getting out of bed from 12 until 5 , when he had free evacuations from his bowels, and fell into a gentle sleep. Pulse moderate, skin cool, tumor evidently diminished. Patient says he is entirely free from pain.

11 A.M. Patient asleep.

Half-past 2 P.M. I called, and thought the patient better than yesterday; the wound has discharged considerably since last evening. I suppose the confinement and accumulation of the matter produced the delirium and other symptoms of irritation. I took off the lower strap, to allow of a free exit for the pus; observed that the granulations were healthy; sponged the wound and tumor; the latter being considerably less than yesterday, and much softer; pulse 104.
11 P.M. Pulse 108 ; in other respects as before by my orders, patient had oranges in the afternoon 27.-7 A.м. Patient has not slept during the night. About 12 he had a very copious evacuation, after which he felt very comfortable; tumor evidently smaller than yesterday; pulse 100 .

28. Patient had evacuations at 3 A.M., since which time he has slept; pulse 125; free from pain.

I P.M. I called, and took off all the straps, and sponged the wound. Discharge healthy, and not profuse. Tumor very soft, and much diminished. I am surprised that the blood continues so long fluid; patient ordered to take more support, as the suppurative stage is established, and I fear exhaustion; pulse 102

29. Pulse 108, jerks. Rheumatic sensations in his limbs and other parts of his body. Respiration quick. Spirits good. Tumor very soft, and the skin over it thin. Discharge from the wound thin, and not so copious.

30. The tumor began to discharge dark blood at ten last night. Pil. Opii prescribed. Rested tolerably well during the night ; in a perspiration this morning; feels quite easy. Poultice continued on the wound. Tongue brown, but moist. Two P.M. I called and pressed the blood from the aneurismal sac, and I was glad to find it had begun to discharge. Ordered a sponge wet with vinegar and water to be laid over the tumor, which now appears a flaccid dark-coloured bag. The patient's pulse is soft and moderately slow, and is better than it has yet been. A light poultice continued on the wound, which looks remarkably well, and discharges healthy pus. No pain. Ten P.м. patient asleep-has taken an opium pill.

July 1. 9 A.M. Skin cool, pulse soft, aneurismal sac entirely collapsed. Sponge with vinegar and water continued. 2 P.M. I saw the patient, and dressed the collapsed sac with lint. The discharge of dark blood still continues, but in small quantities. Pulse moderate. Wound healthy - poultice continued. 9 P.M. Pulse stronger and fuller; has had three evacuations to-day. $\mathbf{P}$. Opii, gr. i. prescribed. Full diet.

July, from 2 to 7 . The wound and bottom of the aneurismal sac (the roof having sloughed away) dressed with lint, and a poultice applied over all; healthy pus discharged. Granulations from both the wound and the sac florid and healthy.

8. The upper ligature came away this morning with the poultice, no force having been ever used to bring it away. Lint and poultice continued.

9. The lower ligature came away; wound healthy, sac filling rapidly; granulations florid.

10 to 12 . No alteration.

13. No change except a little languor from heat. The bed moved into a cooler position. A pint of porter daily ordered.

14 and 15. Doing well.

In about a week afterwards the tumor in the groin had completely closed. After this he was frequently at night attacked with fever, pains in the thigh and leg on the diseased side, and also commencing at the wound, and shooting across the belly; and with lowness of spirits. His appetite and strength, however, daily increased.

In about eight or nine days after the wound had closed, the scab was struck off, and a little matter 
oozed out for several days; but it again closed, and he then felt the fever, \&c. as above described, with sharp pains across the belly. On Thursday, 25th, a quantity of yellow thick matter forced its way out at the part where the operation was performed, and also at the part which had closed below. The discharge continued all night, and he was much easier than before, without fever, pain at lower part of belly, \&c.

On Friday morning a bread and milk poultice was applied, and the discharge continued pretty copious until the following morning. Previous to the breaking of this abscess he had walked a quarter of a mile.

On the 1st of September, the two orifices were closed, but a fresh outlet appeared in the groin, and about a quarter of a pint of matter was discharged through two openings. He says that he feels much easier since this last discharge has taken place. He has very little fever, his appetite is very good, and he increases in strength, but sleeps very little.

The discharge continued freely from the two openings in the groin, but not from the two former places until Saturday, September 3. On that day he walked some distance, drank some wine, and when he returned home, the two orifices, from which the matter had been previously discharged, but which had for some time been closed, again opened and discharged about a quarter of a pint of yellow fetid matter as before. On the same day a diarrhœa took place without any evident cause; and, notwithstanding the administration of numerous remedies, continued to be profuse for seven days; at the end of which time it became less frequent. $\mathrm{He}$ continued in this state, being purged occasionally, once or twice a week. The discharge from the four openings, amounted to about a quarter of a pint daily, until September 23, when another abscess appeared about half an inch above the wound where the operation was performed. On the day following it burst, and discharged nearly half a pint of pus. The surrounding parts appeared somewhat inflamed and indurated. Poultices have been continued over the whole, the Pil. Opii being administered at night; and he is still, notwithstanding the great discharge, able to walk about a little, but complains of frequent pains in the diseased limb, and occasionally, he says, in the bone of the thigh. On walking, the limb swells from the hip down to the ankle. His appetite is very good, he eats five times per day, drinks two pints of porter, and occasionally wine, or wine and water.

In the expectation of inducing a more vigorous state of constitution by the assistance of country air, the patient now left London, and Sir Astley Cooper makes no note of having afterwards heard of him.

\section{CASES OF LACERATION} or

THE POSTERIOR PART

OF THE VAGINA AND CERVIX UTERI,

SUCCESSFULLY TREATED.

BY JONATHAN TOOGOOD, ESQ.

SENIOR SURGEON TO THE BRIDGEWATER INPIRMARY.

IN a paper published by Mr. Birch in the 13th volume of the Medico-Chirurgical Transactions, he states that lacerations of the posterior part of the vagina and cervix uteri always prove fatal. Having met with two cases of very extensive laceration of that part which healed completely in a short time, I think the publication of them may be useful.

CASE I.-Mrs. Collins, a delicate woman of thirty, was seized with uterine hæmorrhage at the seventh month, which recurred slightly twice before the completion of the eighth, when it increased suddenly to an alarming extent. It was then ascertained that the placenta was lying over the os uteri, and immediate delivery was determined on. At ten in the morning of the 19th of April this was effected without any particular difficulty, but with considerable hæmorrhage, which continued after the expulsion of the placenta, to such a degree as to make it advisable to introduce the hand for the purpose of checking it. I then discovered a very extensive laceration of the posterior part of the vagina, which extended to the cervix uteri. Another surgeon, who was present at the delivery, made, at my request, a careful examination, and satisfied himself of the nature and extent of the rupture. The uterus was well contracted. For two hours she remained in a most alarming state, the hæmorrhage continuing, with constant restlessness, fainting, and exhaustion. About half-past twelve she appeared to be rapidly sinking, when a violent fit of vomiting came on, which seemed to relieve her, and she slept for half an hour; but during that time it was often doubtful whether she was still alive. She was quite cold over the whole surface, and this state continued for three hours. Cordials and opium were administered unsparingly, and she gradually became warmer, and now complained of great pain in the belly. She suffered much from exhaustion, but had some sleep during the night.

Second day.- Her countenance was rather improved; the pulse perceptible, but very small. She had occasional sickness, and still some hæmorrhage : complained of excessive exhaustion, but not of noise in the ears, and loss of sight, but never quite fainted. Cordials and opium were given, and perfect quiet enjoined. In the evening reaction commenced; the pulse became full, but easily compressed; the pain in the belly was increased, with feeling of great sinking and weakness. The catheter was passed, and effervescing medicines, with opium, given.

'Third day.- The night was passed without sleep, and the pain in the belly much increased, with restlessness, thirst, full, frequent, weak pulse, sickness, and sinking feeling. The belly was fomented, the catheter passed, and an injection of warm water given; cordials and nutritious food were continued, with perfect quiet. I found her much the same in the evening; but she had slept a good deal at short intervals during the day.

Fourth day.- She had had no sleep during the night, and I found her extremely restless, hot, and flushed in the fac 2 , with a pulse of 149: complaining of extreme weakness, and fainting, with sickness and frequent vomiting: the belly tense, countenance exceedingly anxious, very talkative, and desponding. All the symptoms had become so much worse, that I scarcely hoped to find her alive on my next visit. The cathet $\mathrm{r}$ was passed, a warm water injection thrown $u_{p}$, and an opiate given. 